\title{
Triangle singularities in the production of $X(3872)$
}

\author{
Li-Ping He*, Kevin Ingles, Eric Braaten \\ Department of Physics, The Ohio State University, Columbus, Ohio 43210, USA \\ E-mail: braatendosu.edu, he.1011@buckeyemail.osu.edu, \\ ingles.27@buckeyemail.osu.edu

\section{Jun Jiang} \\ School of Physics, Shandong University, Jinan, Shandong 250100, China \\ E-mail: jiangjun87@sdu.edu.cn
}

\begin{abstract}
If the $X(3872)$ is a loosely bound charm-meson molecule, it can be produced by the creation of its constituents $D^{* 0} \bar{D}^{0}$ or $D^{0} \bar{D}^{* 0}$ at short distances followed by the formation of the bound state from the charm-meson pair. It can also be produced by the creation of $D^{*} \bar{D}^{*}$ at short distances followed by the rescattering of the charm-meson pair into $X(3872) \gamma$ or $X(3872) \pi$ through a triangle loop. The reaction rate for such a process has a triangle singularity, because the three charm mesons that form the triangle loop can all be on shell simultaneously. Triangle singularities produce narrow peaks in the production of $X(3872)$ accompanied by a photon in $e^{+} e^{-}$annihilation, in the production of $X(3872)$ accompanied by a pion in $B$ meson decays, and in the prompt production of $X(3872)$ accompanied by a pion at hadron colliders. The observation of those narrow peaks would prove definitively that $X(3872)$ is a loosely bound charm-meson molecule.
\end{abstract}

\footnotetext{
*** 10th International Workshop on Charm Physics (CHARM2020), ***

*** 31 May - 4 June, 2021 ***

*** Mexico City, Mexico - Online ***
}

* Speaker. 


\section{Introduction}

Since early in this century, dozens of exotic hadrons that contain heavy quarks have been discovered. Understanding these exotic heavy hadrons and predicting others that remain to be discovered are major challenges in particle physics. The first exotic hadron to be discovered was the $X(3872)$ (also known as $\chi_{c 1}(3872)$ ). It was discovered in 2003 by the Belle experiment in exclusive decays of $B^{ \pm}$mesons into $K^{ \pm} X$ through the decay of $X$ into $J / \psi \pi^{+} \pi^{-}$followed by the decay of $J / \psi \rightarrow \mu^{+} \mu^{-}$[1]. The existence of $X$ was quickly verified through inclusive production in $p \bar{p}$ collisions by the CDF experiment at the Tevatron [2]. In 2013, the $J^{P C}$ quantum numbers of $X$ were determined definitively by the LHCb collaboration to be $1^{++}$[3]. The possibilities for the particle structure of $X$ that are compatible with this information include

- the $\chi_{c 1}(2 P)$ charmonium state, whose quark constituents are $c \bar{c}$,

- a compact isospin-1 tetraquark meson, whose diquark constituents are $(c u)(\bar{c} \bar{u})-(c d)(\bar{c} \bar{d})$,

- an isospin-0 charm-meson molecule, whose hadron constituents are $\left(D^{* 0} \bar{D}^{0}+D^{0} \bar{D}^{* 0}\right)+$ $\left(D^{*+} D^{-}+D^{+} D^{*-}\right)$, which correspond to quark constituents $(c \bar{u})(\bar{c} u)+(c \bar{d})(\bar{c} d)$.

The LHCb collaboration has recently made the most precise measurements of the mass of $X$ to date, and they made the first measurements of its width [4,5]. With the line shape of the $X$ in the $J / \psi \pi^{+} \pi^{-}$decay channel modeled by that of a Breit-Wigner resonance, their results for the energy relative to the $D^{* 0} \bar{D}^{0}$ threshold and for the width are

$$
\begin{aligned}
E_{\mathrm{BW}} & =(-0.07 \pm 0.12) \mathrm{MeV}, \\
\Gamma_{\mathrm{BW}} & =(1.19 \pm 0.19) \mathrm{MeV} .
\end{aligned}
$$

The quantum numbers $1^{++}$of $X$ imply that it has an S-wave coupling to $D^{* 0} \bar{D}^{0}$ and $D^{0} \bar{D}^{* 0}$. The charm mesons interact with each other through short-range interactions with a range of order $1 / m$, where $m$ is the pion mass. The small mass difference between the $X$ and $D^{* 0} \bar{D}^{0}$ threshold implies that $X$ has resonant couplings to $D^{* 0} \bar{D}^{0}$ and $\bar{D}^{* 0} D^{0}$ that transforms $X$ into a loosely bound molecule of neutral charm mesons. General principles of quantum mechanics guarantee that $X$ must have universal properties determined by its binding energy [6, 7]. They guarantee that the dominant component of $X$ must be a charm-meson molecule with the particle structure: $X=\left(D^{* 0} \bar{D}^{0}+D^{0} \bar{D}^{* 0}\right) / \sqrt{2}$. However, there are alternative models to the $X$ and no consensus has been reached on its nature [8]. Further studies of its production and its decays are essential to solve this problem.

One way in which the nature of a hadron can be revealed by its production is through triangle singularities. They are kinematic singularities that arise if three virtual particles that form a triangle in a Feynman diagram can all be on their mass shells simultaneously [9, 10]. Studies and applications of triangle singularities can be found in Ref. [11] and references therein. If $X$ is a loosely bound charm-meson molecule, any high-energy process that can create an $D^{*} \bar{D}^{*}$ pair at short distances can produce $X \gamma$ or $X \pi$ with a narrow peak near the $D^{*} \bar{D}^{*}$ threshold due to a charm-meson triangle singularity. In the following sections, we will discuss the effects of triangle singularities on the production of $X \gamma[12,13]$ and $D^{* 0} \bar{D}^{0} \gamma[14]$ in $e^{+} e^{-}$annihilation, on the production of $X \pi$ in decays of $B$ mesons into $K+X \pi$ [15], and on the prompt production of $X$ and a soft pion at hadron colliders [16]. 


\section{2. $X(3872)$ as a weakly bound S-wave molecule and XEFT}

The extremely small energy $E_{X}$ of $X$ relative to the $D^{* 0} \bar{D}^{0}$ threshold in Eq. (1.1a) has dramatic implications for the structure of $X$. If $E_{X}<0, X$ is a bound state whose spatial structure is described by a universal wavefunction $\exp (-r / a) / r$, where $a=1 / \sqrt{2 \mu\left|E_{X}\right|}$ is the S-wave scattering length of $D^{* 0} \bar{D}^{0}$ in the $C=+$ channel and $\mu$ is the reduced mass of $D^{* 0} \bar{D}^{0}$. The mean separation of the constituents is $\langle r\rangle=a / 2$. If the energy $E_{X}$ is identified with the Breit-Wigner energy in Eq. (1.1a), the lower bound on the energy at the $90 \%$ confidence level is $E_{X}>-0.22 \mathrm{MeV}$. Thus the mean separation is larger than $4.8 \mathrm{fm}$. The low-energy scattering of the constituents also has universal aspects determined by $a$ through a simple function of the complex energy $E$ relative to the scattering threshold: $f(E)=1 /[-1 / a+\sqrt{-2 \mu(E+i \varepsilon)}]$.

The universality of S-wave near-threshold resonances provides a basis for a systematically improvable treatment of the $X$ resonance using effective field theory. An appropriate effective field theory was invented by Fleming et al. and named XEFT [17]. It provides a systematically improvable description of the sector of QCD consisting of $D^{*} \bar{D}, D \bar{D}^{*}, D \bar{D} \pi$, or $X$ with total energy near the $D^{*} \bar{D}$ threshold. It can also be applied to the sector consisting of $D^{*} \bar{D}^{*}, D^{*} \bar{D} \pi, D \bar{D}^{*} \pi, D \bar{D} \pi \pi$, or $X \pi$ near the $D^{*} \bar{D}^{*}$ threshold [18]. A Galilean-invariant formulation of XEFT that exploits the approximate conservation of mass in the transitions $D^{*} \leftrightarrow D \pi$ was introduced in Ref. [19] and further developed in Ref. [20].

\section{Charm meson triangle singularity}

If $X$ is a weakly bound charm-meson molecule, it can be produced by the creation of $D^{* 0} \bar{D}^{* 0}, D^{* 0} D^{*-}$, or $D^{*+} \bar{D}^{* 0}$ at short distances followed by the rescattering of the charm-meson pair into $X$ and a photon or pion through a triangle loop at longer distances. Fig. 1 shows the Feynman diagram for the production of $X$ and a $\pi^{0}$ from rescattering of $D^{* 0} \bar{D}^{* 0}$. There is a triangle singularity in the production rate if the three charm mesons in the loop can be on-shell simultaneously. The two charm mesons attached to the $X$ can be on shell in
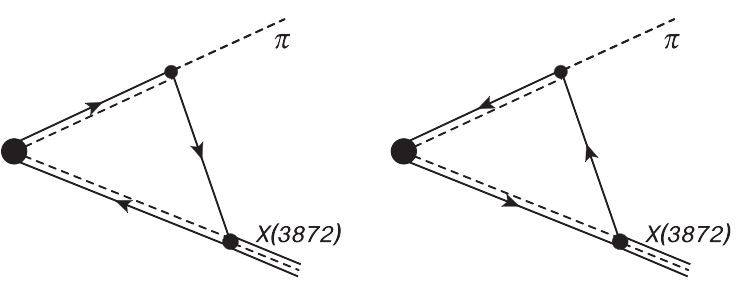

Figure 1: Feynman diagram for $D^{* 0} \bar{D}^{* 0}$ created at a point to rescatter into $X \pi^{0}$. The $X$ is represented by a triple (solid+dashed+solid) line. The $D$ and $\bar{D}$ are represented by solid lines. The $D^{*}$ and $\bar{D}^{*}$ are represented by double (solid+dashed) lines. The $\pi$ is represented by a dashed line.

the limit of zero $X$ binding energy $\left|E_{X}\right|$ and zero $D^{*}$ decay width $\Gamma_{*}$. The third charm meson in the triangle can be made on shell by adjusting the energy of the final-state photon or pion. The amplitude includes a logarithmic term of the form

$$
F(W)=\log \frac{\sqrt{2 \mu E_{X}+i \mu \Gamma_{*}}+\sqrt{M_{*}\left(W+i \Gamma_{*}\right)}+\left(M_{*} / M_{X}\right) q}{\sqrt{2 \mu E_{X}+i \mu \Gamma_{*}}+\sqrt{M_{*}\left(W+i \Gamma_{*}\right)}-\left(M_{*} / M_{X}\right) q},
$$

where $W$ is the center-of-mass $(\mathrm{CM})$ energy relative to the threshold of $D^{*} \bar{D}^{*}, M_{*}$ and $M_{X}$ are the masses of $D^{*}$ and $X$, and $q$ is the relative momentum of $X$ and the outgoing photon or pion. The 
triangle singularity arises from the logarithm in Eq. (3.1). The denominator of the argument of the logarithm vanishes at a complex energy that approaches the real axis in the limits $\Gamma_{*} \rightarrow 0$ and $E_{X} \rightarrow 0$. In these limits, the denominator is zero at the energy for which $\sqrt{M_{*} W}=\left(M_{*} / M_{X}\right) q(W)$, where $q(W)$ is a function of $W$. We denote $\delta_{0 \pi}$ as the mass difference between $D^{*}$ and $D^{0} \pi$ and $\delta_{0 \gamma}$ as the mass difference between $D^{* 0}$ and $D^{0} \gamma$. The energy $W_{\triangle}$ at which the triangle singularity occurs can be obtained by solving the equation $\sqrt{M_{*} W}=\left(M_{*} / M_{X}\right) q(W)$ :

$$
W_{\triangle} \begin{cases}=(m / 2 M) \delta_{0 \pi^{ \pm}}=0.2 \mathrm{MeV}, & \text { for } X \pi^{ \pm} \text {production, } \\ =(m / 2 M) \delta_{0 \pi^{0}}=0.3 \mathrm{MeV}, & \text { for } X \pi^{0} \text { production, } \\ \approx\left(M_{*} / M_{X}^{2}\right) \delta_{0 \gamma}^{2}=2.7 \mathrm{MeV}, & \text { for } X \gamma \text { production, }\end{cases}
$$

where $M$ is the mass of $D^{0}$. The triangle singularity produces a double-log divergence at $W_{\triangle}$ in the reaction rate. Taking the physical decay width of $D^{*}$ and a nonzero binding energy of the $X$ into account, the triangle singularity produces a narrow peak in the reaction rate at an energy close to $W_{\triangle}$

\section{Production of $X+\gamma$ in $e^{+} e^{-}$annihilation}

The quantum numbers $1^{++}$of the $X$ imply that $X \gamma$ can be produced by $e^{+} e^{-}$annihilation into a virtual photon. The virtual photon can create $D^{* 0} \bar{D}^{* 0}$ at short distances in a P-wave channel, and the charm-meson pair can subsequently rescatter into $X \gamma$. The
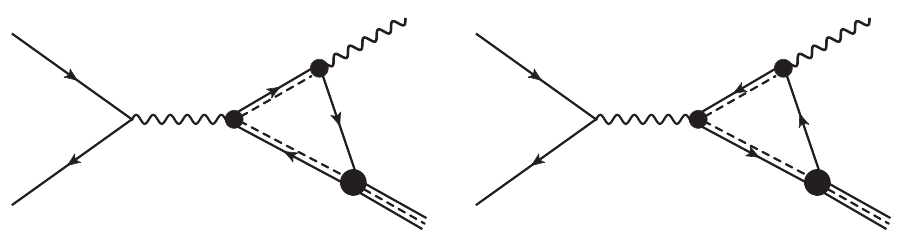

Figure 2: Feynman diagrams for $e^{+} e^{-} \rightarrow \gamma X$ from rescattering of $D^{* 0} \bar{D}^{* 0}$. production of $X \gamma$ in $e^{+} e^{-}$annihilation near the $D^{* 0} \bar{D}^{* 0}$ threshold was first discussed by Dubynskiy and Voloshin [21]. They calculated the absorptive contribution to the cross section from $e^{+} e^{-}$annihilation into onshell charm mesons $D^{* 0} \bar{D}^{* 0}$ followed by their rescattering into $X \gamma$. They predicted that the cross section has a narrow peak only a few $\mathrm{MeV}$ above the $D^{* 0} \bar{D}^{* 0}$ threshold. In retrospect, their narrow peak comes from a triangle singularity. In Refs. [12, 13], we calculated the cross section for $e^{+} e^{-} \rightarrow X \gamma$ in the energy region near the $D^{* 0} \bar{D}^{* 0}$ threshold, including the dispersive contribution as well as the absorptive contribution. The corresponding Feynman diagrams are shown in Fig. 2. We also predicted the normalization of the cross section for $e^{+} e^{-} \rightarrow X \gamma$ using a fit to the cross section for $e^{+} e^{-} \rightarrow D^{*+} D^{*-}$ by Uglov et al. [22]. The cross section has a narrow peak at an energy 2.2 $\mathrm{MeV}$ above the $D^{* 0} \bar{D}^{* 0}$ threshold as shown by the solid lines in Fig. 3 . The peak is caused by a charm-meson triangle singularity. The comparison between the full cross section and the absorptive contribution is shown in Fig. 3. The absorptive contribution is not a good approximation near the triangle singularity region. The position of the peak in the full cross section is much less sensitive to the binding energy, and the height of the peak is significantly larger. If the $X$ is observed in the decay mode $J / \psi \pi^{+} \pi^{-}$, the cross section must be multiplied by the branching fraction $\mathrm{Br}$ for the bound state to decay into $J / \psi \pi^{+} \pi^{-}$. The loose lower bound $\mathrm{Br}>4 \%$ and the upper bound $\mathrm{Br}<33 \%$ on that branching fraction were derived in Ref. [23]. 
Shortly before our work, Guo pointed out that any high-energy process that can create $D^{* 0} \bar{D}^{* 0}$ at short distances in an $\mathrm{S}$-wave channel will produce $X \gamma$ with a narrow peak near the $D^{* 0} \bar{D}^{* 0}$ threshold due to a charmmeson triangle singularity [24]. The cross sections for $e^{+} e^{-} \rightarrow \pi^{0} \gamma X$ and $p \bar{p} \rightarrow \gamma X$ have been estimated by Sakai, Jing and Guo [25].

The production of $X \gamma$ in $e^{+} e^{-}$annihilation has been studied by the BESIII collaboration [26, 27]. However, the cross section was not measured at energies near 4.016 $\mathrm{GeV}$, which is where the narrow peak from the charm-meson triangle singularity is predicted to appear. The cross section near the peak is large enough that it may be observed in the future by the BESIII experiment.

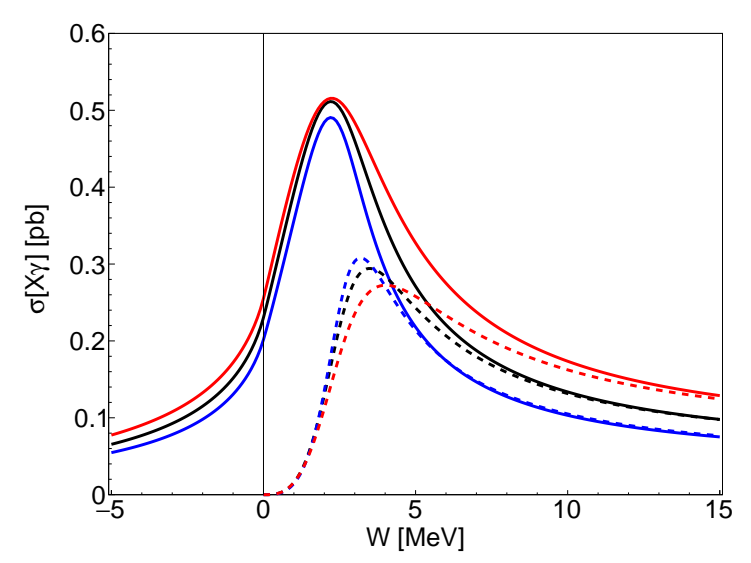

Figure 3: Cross section with full amplitude (solid lines) and with absorptive part only (dashed lines) for $e^{+} e^{-} \rightarrow X(3872) \gamma$ as functions of the CM energy $W$ relative to the $D^{* 0} \bar{D}^{* 0}$ threshold. The three curves in order of decreasing cross sections are for binding energies $\left|E_{X}\right|=0.30 \mathrm{MeV}$ (red), $0.17 \mathrm{MeV}$ (black), and $0.10 \mathrm{MeV}$ (blue).

\section{Production of $\boldsymbol{D}^{* 0} \overline{\boldsymbol{D}}^{0} \boldsymbol{\gamma}$ in $\boldsymbol{e}^{+} \boldsymbol{e}^{-}$ annihilation}

Since $D^{* 0} \bar{D}^{0}$ are constituents of $X$, the annihilation of $e^{+} e^{-}$can also produce $D^{* 0} \bar{D}^{0}+\gamma$. In Ref. [14], we have studied the effects of the charm-meson triangle singularity on the cross section for $e^{+} e^{-}$annihilation into $D^{* 0} \bar{D}^{0}+\gamma$ just above the $D^{* 0} \bar{D}^{* 0}$ threshold. The matrix element for this reaction is the sum of the tree diagram in Fig. 4 and a

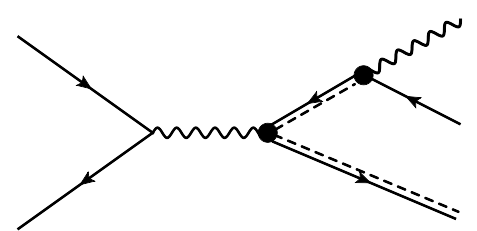

Figure 4: Tree diagram for $e^{+} e^{-} \rightarrow D^{* 0} \bar{D}^{0}+\gamma$. pair of loop diagrams with a charm-meson triangle. The loop diagrams can be obtained from Fig. 2 by replacing the $X$ by $D^{* 0} \bar{D}^{0}$. The differential cross section $d \sigma / d u d t$ is a function of the squared invariant masses $u$ of $D^{* 0} \bar{D}^{0}$ and $t$ of $\bar{D}^{0} \gamma$ as well as the squared CM energy $s$.

There is triangle singularity from the region where the three charm mesons that form the loop are all on their mass shells. The range of $s$ for the triangle singularity is $4 M_{*}^{2}<s \leq s_{\triangle}$, where $s_{\triangle}=4 M_{*}^{2}+\left(M_{*} / M\right)\left(M_{*}-M\right)^{2}$ is the squared invariant mass when $u=\left(M_{*}+M\right)^{2}$. The triangle singularity solution for $u$ is a function of $s$ :

$$
u_{\triangle}(s)=\left(M_{*}+M\right)^{2}+\left[\left(M_{*}-M\right) \sqrt{s}-\left(M_{*}+M\right) \sqrt{s-4 M_{*}^{2}}\right]^{2} /\left(4 M_{*}^{2}\right) .
$$

In the limit of zero $D^{* 0}$ width, the triangle singularity leads to a double-logarithmic divergence, which produces a narrow band in the squared invariant mass $u$ of $D^{* 0} \bar{D}^{0}$ in the Dalitz plot. An interesting aspect of this reaction is a cancellation pointed out by Schmid [28]. In the differential cross section integrated over $t$, the double-logarithmic divergence is canceled by the interference between 
the tree diagram and the loop diagrams. We verified that the cancellation leaves a singlelogarithmic divergence as shown previously by Anisovitch and Anisovitch [29].

At the physical value of the $D^{* 0}$ width, the narrow band in the Dalitz plot reduces to a shoulder. Thus the triangle singularity cannot be observed directly as a peak in a differential cross section as a function of $u$. It may however be observed indirectly as a local minimum in the $t$ distribution for events with $u$ below the triangle singularity, as illustrated in Fig. 5. The minimum is produced by the Schmid cancellation between the triangle loop diagrams and the tree diagram. The observation of this minimum would support the identification of $X$ as a loosely bound charm meson molecule.

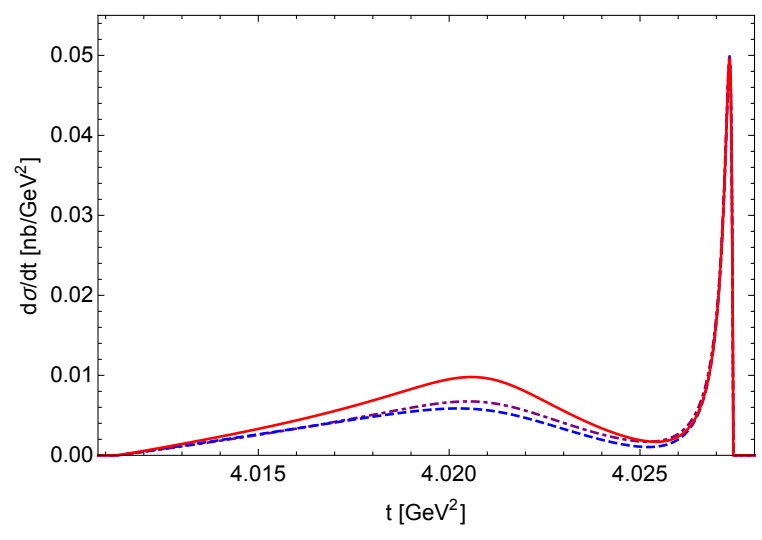

Figure 5: Differential cross section $d \sigma / d t$ for producing $D^{* 0} \bar{D}^{0}+\gamma$ integrated over $u<u_{\triangle}(s)$ as a function of $t$ at $\sqrt{s}=4014.7 \mathrm{MeV}$. The binding energy and width of $X$ are $\left|E_{X}\right|=0.05 \mathrm{MeV}, \Gamma_{X}=\Gamma_{* 0}$ (solid red curve), $\left|E_{X}\right|=0.10 \mathrm{MeV}, \Gamma_{X}=\Gamma_{* 0}$ (dashed blue curve), and $\left|E_{X}\right|=0.05 \mathrm{MeV}, \Gamma_{X}=2 \Gamma_{* 0}$ (dot-dashed purple curve).

\section{Production of $X+\pi$ from $B$ meson decay}

Another process that can produce $D^{*} \bar{D}^{*}$ at short distances is $B$ meson decay. In Ref. [15], we studied the production of $X+\pi$ in exclusive decays $B \rightarrow K X \pi$. This reaction can proceed through the decay of $B$ at short distances into $K$ plus a $D^{*} \bar{D}^{*}$ pair with small relative momentum followed by the rescattering of $D^{*} \bar{D}^{*}$ into $X \pi$. We used a precise isospin analysis of the decays $B \rightarrow$ $K D^{(*)} \bar{D}^{(*)}$ by Poireau and Zito [30] to obtain approximations for the short-distance amplitudes for these decays. We used XEFT to calculate the amplitude for the rescattering of $D^{*} \bar{D}^{*}$ into $X \pi$. The distributions of the kinetic energy $E_{X \pi}$ of $X$ and $\pi$ in the $X \pi \mathrm{CM}$ frame are illustrated in Fig. 6. There is a narrow peak near the $D^{*} \bar{D}^{*}$ threshold from a charm-meson triangle singularity. For the decays $B \rightarrow K X \pi^{0}$, the peak in $E_{X \pi}$ is predicted to be near 7.3 MeV. For the decays $B \rightarrow K X \pi^{ \pm}$, the peak is predicted to be near $6.1 \mathrm{MeV}$.

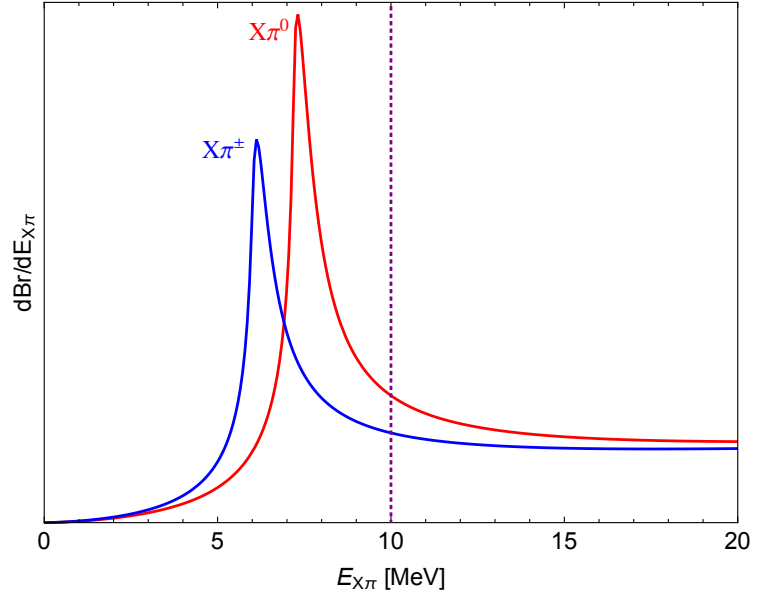

Figure 6: Differential branching fractions $d \mathrm{Br} / d E_{X} \pi$ for the decays $B \rightarrow K X \pi^{0}$ and $B \rightarrow K X \pi^{ \pm}$as functions of the kinetic energy $E_{X \pi}$. The binding energy of the $X$ is $0.17 \mathrm{MeV}$. The region of validity of XEFT extends out to about the vertical dotted line at $E_{X \pi}=10 \mathrm{MeV}$. The normalizations of the curves are arbitrary. The relative normalizations of the $X \pi^{0}$ and $X \pi^{ \pm}$curves are chosen so their extrapolations to large $E_{X \pi}$ are equal. 
Our estimates of the branching fractions for decays of $B$ into $K$ plus $X \pi$ in the peak from the charm-meson triangle singularity suggest that it may be possible to observe the narrow peak from the charm-meson triangle singularity. The observation of a peak in the $X \pi$ invariant mass distribution near the $D^{*} \bar{D}^{*}$ threshold would provide strong support for the identification of $X$ as a loosely bound charm-meson molecule and present a serious challenge to other models.

Other subsequent works related to the triangle singularity in $B$ meson decays are Refs. [31, 32, 33].

\section{Production}

\section{of $X+\pi$ at hadron colliders}

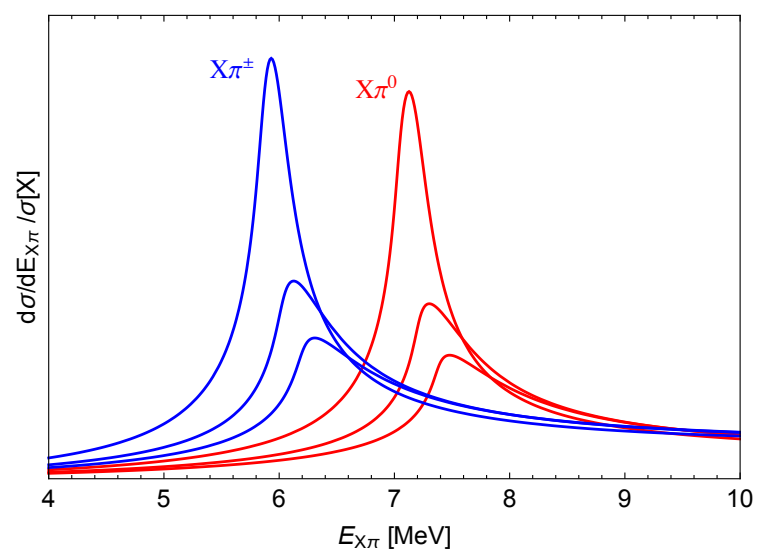

Figure 7: Differential cross sections $d \sigma / d E_{X \pi} / \sigma[X]$ as functions of the kinetic energy $E_{X \pi}$ for $X \pi^{0}$ and for $X \pi^{ \pm}$summed over \pm . In order of decreasing height, the curves are for binding energies $\left|E_{X}\right|=0,0.17$, and $0.34 \mathrm{MeV}$. The scale on the vertical axis is arbitrary, but the relative normalizations of the curves are determined.

The prompt production of $X+\pi^{ \pm}$can be studied at a hadron collider. The charged pion provides a clean signature for this new production mechanism. A large number of $D^{*} \bar{D}^{*}$ pairs are produced promptly at the primary vertex. In Ref. [16], we studied the inclusive prompt production of $X+\pi$ through the triangle loops shown in Fig. 1. The differential cross section for the production of $X \pi^{+}$from rescattering of $D^{*+} \bar{D}^{* 0}$ is illustrated in Figure 7. There is a narrow peak from a charm-meson triangle singularity at a kinetic energy $E_{X \pi}$ about $6.1 \mathrm{MeV}$ above the $X \pi^{+}$threshold.

The D0 collaboration has reported studies of the production of $X \pi^{ \pm}$in both prompt production and $b$-hadron decay in $p \bar{p}$ collisions at the Tevatron [34]. They measured the production rate as functions of the kinetic energy $T(X \pi)$ of $X \pi^{ \pm}$in their $\mathrm{CM}$ frame. For the prompt production of $X \pi^{ \pm}$, they observed $18 \pm 16 X \pi$ events with $T(X \pi)$ below $11.8 \mathrm{MeV}$. The expected number from combining $X$ with a random pion in the event was 6. They concluded that there is no evidence for an enhancement in the prompt production of $X$ accompanied by a soft $\pi^{ \pm}$as expected from the triangle singularity. For the production of $X \pi^{ \pm}$from $b$-hadron decay, they observed $27 \pm 12$ events with $T(X \pi)$ below

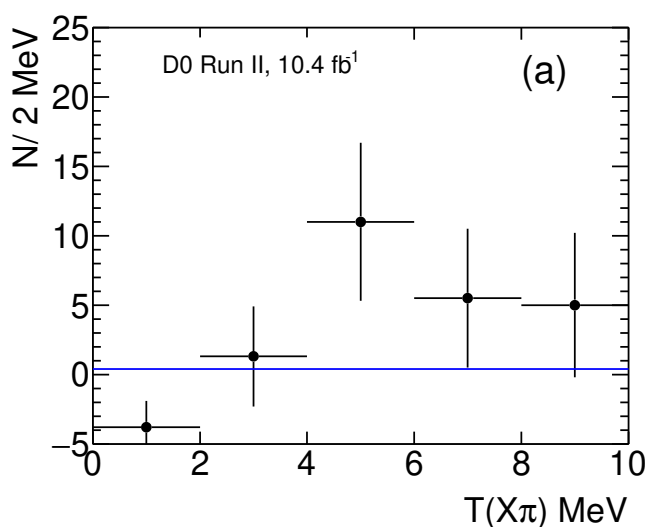

Figure 8: Data from the D0 collaboration on $X \pi^{ \pm}$ production from $b$-hadron decay in $p \bar{p}$ collisions at the Tevatron as a function of the kinetic energy $T(X \pi)$ [34].

11.8 MeV. The expected number from combining $X$ with a random pion in the event is 2 . They 
concluded that there is no "significant" evidence for the production of $X$ accompanied by a soft $\pi^{ \pm}$ as expected from the triangle singularity. They did however observe a small excess in the small $T(X \pi)$ region with a significance of $2 \sigma$, as shown in Fig. 8. The excess is largest in the bin of $T(X \pi)$ between 4 and $6 \mathrm{MeV}$. It is tempting to regard this excess as the first hint of the peak from the triangle singularity.

\section{Summary and outlook}

In summary, the charm meson triangle singularity produces narrow peaks in the production of $X+\gamma / \pi$ just above $D^{*} \bar{D}^{*}$ threshold. The peak is near $2.2 \mathrm{MeV}$ above $D^{* 0} \bar{D}^{* 0}$ threshold for $X \gamma, 6.1$ $\mathrm{MeV}$ above $X \pi^{ \pm}$threshold for $X \pi^{ \pm}$, and $7.3 \mathrm{MeV}$ above $X \pi^{0}$ threshold for $X \pi^{0}$.

There are several high energy physics experiments that could observe the peaks in the production of $X \gamma$ or $X \pi$ coming from triangle singularities. The narrow peak in the production of $X \gamma$ from a triangle singularity in $e^{+} e^{-}$annihilation could be observed by the BESIII detector. They may also be able to observe the interference effect from the Schmid cancellation. The Belle collaboration has studied the decay of $B^{0} \rightarrow K^{+} X \pi^{-}$, and they did not observe any peak in the $X \pi^{-}$distribution near the threshold [35]. The Belle II experiment will accumulate about 50 times the data of the Belle experiment, so they may be able to observe the peak from the triangle singularity. In a study of $X \pi^{ \pm}$production from inclusive $b$-hadron decay in $p \bar{p}$ collisions, the D0 collaboration has observed a small excess in the region of $X \pi^{ \pm}$kinetic energy near $5 \mathrm{MeV}$ with a significance of $2 \sigma$. This excess may be a first hint of the peak from a triangle singularity. Studies of $X \pi^{ \pm}$production in $p p$ collisions at the Large Hadron Collider by the ATLAS, CMS, and LHCb experiments would benefit from much larger data sets. The observation of narrow peaks in the production of $X \gamma$ or $X \pi$ from triangle singularities would provide strong support for the identification of the $X$ as a loosely bound charm-meson molecule.

\section{Acknowledgements}

This work was supported in part by the U.S. Department of Energy under Grant No. DESC0011726, the National Natural Science Foundation of China under Grant No. 11905112, and the Natural Science Foundation of Shandong Province under Grant No. ZR2019QA012. L.-P. H. was supported in part by a Presidential Fellowship from Ohio State University.

\section{References}

[1] S.K. Choi et al. [Belle Collaboration], Observation of a narrow charmonium-like state in exclusive $B^{ \pm} \rightarrow K^{ \pm} \pi^{+} \pi^{-} J / \psi$ decays, Phys. Rev. Lett. 91, 262001 (2003) [hep-ex/0309032].

[2] D. Acosta et al. [CDF Collaboration], Observation of the narrow state $X(3872) \rightarrow J / \psi \pi^{+} \pi^{-}$in $\bar{p} p$ collisions at $\sqrt{s}=1.96 \mathrm{TeV}$, Phys. Rev. Lett. 93, 072001 (2004) [hep-ex/0312021].

[3] R. Aaij et al. [LHCb], Determination of the $X$ (3872) meson quantum numbers, Phys. Rev. Lett. 110, 222001 (2013) [arXiv:1302.6269].

[4] R. Aaij et al. [LHCb], Study of the lineshape of the $\chi_{c 1}$ (3872) state, Phys. Rev. D 102, 092005 (2020) [arXiv:2005.13419]. 
[5] R. Aaij et al. [LHCb], Study of the $\psi_{2}(3823)$ and $\chi_{c 1}$ (3872) states in $B^{+} \rightarrow\left(J / \psi \pi^{+} \pi^{-}\right) K^{+}$decays, JHEP 08, 123 (2020) [arXiv:2005.13422].

[6] E. Braaten and H. W. Hammer, Universality in few-body systems with large scattering length, Phys. Rept. 428, 259-390 (2006) [arXiv:cond-mat/0410417].

[7] E. Braaten and M. Kusunoki, Low-energy universality and the new charmonium resonance at 3870-MeV, Phys. Rev. D 69, 074005 (2004) [arXiv:hep-ph/0311147].

[8] N. Brambilla, S. Eidelman, C. Hanhart, A. Nefediev, C. P. Shen, C.E. Thomas, A. Vairo and C.Z. Yuan, The $X Y Z$ states: experimental and theoretical status and perspectives, Phys. Rept. 873, 1-154 (2020) [arXiv:1907.07583].

[9] R. Karplus, C. M. Sommerfield and E. H. Wichmann, Spectral Representations in Perturbation Theory. 1. Vertex Function, Phys. Rev. 111, 1187-1190 (1958).

[10] L. D. Landau, On analytic properties of vertex parts in quantum field theory, Nucl. Phys. 13, no.1, 181-192 (1959).

[11] F. K. Guo, X. H. Liu and S. Sakai, Threshold cusps and triangle singularities in hadronic reactions, Prog. Part. Nucl. Phys. 112, 103757 (2020) [arXiv:1912.07030].

[12] E. Braaten, L. P. He and K. Ingles, Triangle Singularity in the Production of X(3872) and a Photon in $e^{+} e^{-}$Annihilation, Phys. Rev. D 100, 031501 (2019) [arXiv:1904.12915].

[13] E. Braaten, L. P. He and K. Ingles, Production of $X(3872)$ and a Photon in $e^{+} e^{-}$Annihilation, Phys. Rev. D 101, 014021 (2020) [arXiv:1909.03901].

[14] E. Braaten, L. P. He, K. Ingles and J. Jiang, Charm-meson triangle singularity in $e^{+} e^{-}$annihilation into $D^{* 0} \bar{D}^{0}+\gamma$, Phys. Rev. D 101, 096020 (2020) [arXiv:2004.12841].

[15] E. Braaten, L. P. He and K. Ingles, Production of $X(3872)$ Accompanied by a Pion in $B$ Meson Decay, Phys. Rev. D 100, 074028 (2019) [arXiv:1902.03259].

[16] E. Braaten, L. P. He and K. Ingles, Production of $X(3872)$ Accompanied by a Soft Pion at Hadron Colliders, Phys. Rev. D 100, 094006 (2019) [arXiv:1903.04355].

[17] S. Fleming, M. Kusunoki, T. Mehen and U. van Kolck, Pion interactions in the $X(3872)$, Phys. Rev. D 76, 034006 (2007) [arXiv:hep-ph/0703168].

[18] E. Braaten, H. W. Hammer and T. Mehen, Scattering of an Ultrasoft Pion and the X(3872), Phys. Rev. D 82, 034018 (2010) [arXiv:1005.1688].

[19] E. Braaten, Galilean-invariant effective field theory for the $X(3872)$, Phys. Rev. D 91, 114007 (2015) [arXiv:1503.04791].

[20] E. Braaten, L. P. He and J. Jiang, Galilean-invariant effective field theory for the $X(3872)$ at next-to-leading order, Phys. Rev. D 103, 036014 (2021) [arXiv:2010.05801].

[21] S. Dubynskiy and M. B. Voloshin, $e^{+} e^{-} \rightarrow \gamma X(3872)$ near the $D^{*} \bar{D}^{*}$ threshold, Phys. Rev. D 74, 094017 (2006) [arXiv:hep-ph/0609302].

[22] T. V. Uglov, Y. S. Kalashnikova, A. V. Nefediev, G. V. Pakhlova and P. N. Pakhlov, Exclusive open-charm near-threshold cross sections in a coupled-channel approach, JETP Lett. 105, 1-7 (2017) [arXiv:1611.07582].

[23] E. Braaten, L. P. He and K. Ingles, Branching Fractions of the $X(3872)$, [arXiv:1908.02807]. 
[24] F. K. Guo, Novel Method for Precisely Measuring the $X$ (3872) Mass, Phys. Rev. Lett. 122, 202002 (2019) [arXiv:1902.11221].

[25] S. Sakai, H. J. Jing and F. K. Guo, Possible precise measurements of the $X(3872)$ mass with the $e^{+} e^{-} \rightarrow \pi^{0} \gamma X(3872)$ and $p \bar{p} \rightarrow \gamma X(3872)$ reactions, Phys. Rev. D 102, 114041 (2020) [arXiv:2008.10829].

[26] M. Ablikim et al. [BESIII], Observation of $e^{+} e \rightarrow \gamma X(3872)$ at BESIII, Phys. Rev. Lett. 112, 092001 (2014) [arXiv:1310.4101].

[27] M. Ablikim et al. [BESIII], Study of $e^{+} e^{-} \rightarrow \gamma \omega J / \psi$ and Observation of $X(3872) \rightarrow \omega J / \psi$, Phys. Rev. Lett. 122, 232002 (2019) [arXiv:1903.04695].

[28] C. Schmid, Final-State Interactions and the Simulation of Resonances, Phys. Rev. 154, 1363 (1967)

[29] A. V. Anisovich and V. V. Anisovich, Rescattering effects in three particle states and the Schmid theorem, Phys. Lett. B 345, 321-324 (1995).

[30] V. Poireau and M. Zito, A precise isospin analysis of $B \rightarrow \bar{D}^{(*)} D^{(*)} K$ decays, Phys. Lett. B 704, 559-565 (2011) [arXiv:1107.1438].

[31] S. X. Nakamura, Triangle singularity appearing as an $X(3872)$-like peak in $B \rightarrow\left(J / \psi \pi^{+} \pi^{-}\right) K \pi$, Phys. Rev. D 102, 074004 (2020) [arXiv:1912.11830].

[32] S. Sakai, E. Oset and F.K. Guo, Triangle singularity in the $B^{-} \rightarrow K^{-} \pi^{0} X(3872)$ reaction and sensitivity to the $X$ (3872) mass, Phys. Rev. D 101, 054030 (2020) [arXiv:2002.03160].

[33] R. Molina and E. Oset, Triangle singularity in $B^{-} \rightarrow K^{-} X(3872) ; X \rightarrow \pi^{0} \pi^{+} \pi^{-}$and the X(3872) mass, Eur. Phys. J. C 80, 451 (2020) [arXiv:2002.12821].

[34] V. M. Abazov et al. [D0], Studies of $X(3872)$ and $\psi(2 S)$ production in $p \bar{p}$ collisions at $1.96 \mathrm{TeV}$, Phys. Rev. D 102, 072005 (2020) [arXiv:2007.13420].

[35] A. Bala et al. [Belle], Observation of $\mathrm{X}(3872)$ in $\mathrm{B} \rightarrow \mathrm{X}(3872) \mathrm{K} \pi$ decays, Phys. Rev. D 91, 051101 (2015) [arXiv:1501.06867]. 